\title{
Saciedade subjetiva, aceitação sensorial e aspectos tecnológicos de salsicha com adição de fibra de trigo
}

\author{
Subjective satiety, sensory acceptance and technological aspects of \\ frankfurter sausage with added wheat fibre
}

\begin{abstract}
Kátia Helena Terríbille Borrajo ${ }^{1 *}$, César Gonçalves de Lima1, Marco Antonio Trindade ${ }^{1}$
${ }^{1}$ Universidade de São Paulo (USP), Faculdade de Zootecnia e Engenharia de Alimentos, Departamento de Engenharia de Alimentos, Pirassununga/SP - Brasil
\end{abstract}

\section{${ }^{*}$ Corresponding Author}

Kátia Helena Terríbille Borrajo, Universidade de São Paulo (USP), Faculdade de Zootecnia e Engenharia de Alimentos, Departamento de Engenharia de Alimentos, Avenida Duque de Caxias Norte, 225, CEP: 13635-900, Pirassununga/SP - Brasil, e-mail: katiaborrajo@usp.br

Cite as: Subjective satiety, sensory acceptance and technological aspects of frankfurter sausage with added wheat fibre. Braz. J. Food Technol., v. 19, e2016008, 2016.

Received: Jan. 19, 2016; Accepted: July 25, 2016

\section{Resumo}

O objetivo deste trabalho foi a elaboração de salsichas com diferentes concentrações de fibra de trigo e a avaliação de suas características tecnológicas, aceitação sensorial e saciedade subjetiva causada por sua ingestão. Foram produzidas salsichas com adição de zero (Controle), 1,5\% e 3\% de fibra de trigo. Os aspectos tecnológicos avaliados foram: composição centesimal (teores de proteínas, lipídios, umidade e cinzas), valor de $\mathrm{pH}$, textura instrumental, cor objetiva, estabilidade de emulsão e rendimento do processo. Para análise sensorial foi realizado teste afetivo de aceitação com 60 consumidores que avaliaram os atributos sabor, textura, suculência e aceitação global, utilizando escala hedônica de 9 pontos. Para a análise de saciedade, 30 consumidores relataram, em uma escala visual analógica, a sensação de fome sentida antes, imediatamente depois e a cada 30 minutos após comerem as salsichas, durante 2 horas. Os resultados demonstraram que, exceto para a luminosidade $\left(L^{*}\right)$, as salsichas com adição de fibras (1,5\% e 3\%) não diferiram em nenhum dos aspectos tecnológicos em relação à amostra Controle. Na avaliação sensorial, as salsichas com 3\% de fibra foram consideradas mais suculentas e tiveram melhor aceitação global $(p<0,05)$ que as com $1,5 \%$, ambas não diferindo da amostra Controle. A aceitação dos atributos sabor e textura não diferiu entre todas as amostras. Em relação à avaliação da saciedade, não houve diferença entre as amostras, ou seja, a adição de até $3 \%$ de fibra de trigo nas salsichas não foi suficiente para promover maior saciedade nos consumidores em comparação com a salsicha sem adição de fibra. Pode-se concluir que, apesar de não ter sido observada maior sensação de saciedade pela adição de fibra de trigo, salsichas contendo 1,5\% e 3\% desta fibra podem ser produzidas sem prejuízo das características tecnológicas e com boa aceitação sensorial, podendo favorecer o aumento do consumo de fibras pela população.

Palavras-chave: Impulso alimentar; Dextrina resistente; Produtos cárneos; Obesidade.

\section{Summary}

The objective of this work was to elaborate frankfurter sausages with different wheat fibre concentrations and evaluate their technological characteristics, sensory acceptance and the subjective satiety caused by their ingestion. Sausages were produced with the addition of zero (control), 1.5 and $3 \%$ of wheat fibre. The technological aspects evaluated were: proximate composition (protein, lipid, moisture and ash), pH value, instrumental texture, objective colour, emulsion stability and process yield. For the sensory evaluation an affective acceptance test was carried out with 60 consumers who evaluated the flavour, texture, juiciness and overall acceptance using a 9-point hedonic scale. For the satiety analysis, 30 consumers reported the hunger sensation felt before, immediately after and every 30 minutes after eating the sausages, for a period of 2 hours, using a visual analogical scale. The results showed that, except for lightness $\left(L^{*}\right)$, the sausages with added fibres $(1.5$ and $3 \%)$ did not differ from the control in any of the technological aspects. In the sensory evaluation, the sausages with $3 \%$ fibre 
were considered juicier and had better overall acceptance $(p<0.05)$ than those with $1.5 \%$, and neither differed from the control. The acceptance of the attributes of flavour and texture was the same for all the samples. With respect to satiety, there was no difference amongst the samples, i.e., the addition of up to $3 \%$ wheat fibre to the sausages was not sufficient to promote greater consumer satiety when compared to the sausage without added fibre. It can be concluded that, although no greater satiety was observed due to the addition of wheat fibre, sausages containing 1.5 to $3 \%$ of this fibre could be produced without prejudicing the technological characteristics and with good sensory acceptance, thus favouring an increase in fibre intake by the population.

Keywords: Food impulse; Resistant dextrin; Meat products; Obesity.

\section{Introdução}

No Brasil, assim como em outros países, observa-se um aumento da prevalência de obesidade, que está estritamente relacionada com mudanças no estilo de vida e nos hábitos alimentares (WANG et al., 2002). Segundo o IBGE (2011), 68\% da população brasileira consome quantidade de fibras abaixo dos valores recomendados e esta mesma pesquisa mostra que dentre os alimentos mais consumidos encontram-se os de preparo rápido, como a salsicha, que merece destaque, pois pertence ao grupo de frios e embutidos no qual se observou aumento de $67,6 \%$ no volume de vendas nesse período.

Uma população que consome dietas ricas em fibra alimentar tem menor incidência de doenças crônicas e degenerativas (RANINEN et al., 2011), pois o consumo elevado dessas fibras está associado à prevenção e tratamento de problemas gastrintestinais (PETRUZZIELLO et al., 2006), diabetes (MONTONEN et al., 2003), obesidade (BIRKETVEDT et al., 2005) e doenças coronárias (LIU et al., 1999). Além de todos estes benefícios, o consumo de fibras alimentares também tem relação com o auxílio no gerenciamento de peso através de diversos mecanismos, incluindo a capacidade da fibra de promover saciedade.

O desenvolvimento de alimentos que forneçam "saciedade" ou "satisfação da fome" é um dos desafios da indústria de alimentos, e a habilidade para medir essa saciedade é de interesse de pesquisadores e produtores. A fome, o impulso para ingerir alimentos para saciá-la e a sensação de saciedade são causados por vários mecanismos de sinalização, com participação de diversas substâncias, por exemplo, a ação da grelina conforme a distensão das paredes do estômago, as do glucagon 1 e 2, liberados pelas células endócrinas do intestino na corrente sanguínea em resposta à ingestão de açúcares, estimulando a produção da insulina (INUI et al., 2004), da colecistocinina (CCK) liberada no duodeno em resposta à presença de proteínas e gorduras (HALPERN et al., 2004), entre outros mecanismos. Pelo menos três métodos têm sido utilizados para medir a saciedade em seres humanos: por meio de indicadores fisiológicos; pela quantificação de ingestão de alimentos (volume, peso, calorias); e avaliações subjetivas (NAZARE et al., 2011; FLINT et al.,
2000), utilizando, por exemplo, a escala visual analógica aplicada no presente trabalho.

Diferentes fibras têm sido adicionadas em produtos cárneos como a de trigo, aveia e inulina em mortadela e salsichas, carragenina e carragena com pectina em salsichas tipo Frankfurters, goma guar e goma xantana em salsichas, amido e carragena em presunto cozido de peru (HUANG et al., 2011; CANDOGAN; KOLSARICI, 2003; GARCIA-CRUZ et al., 1996). A fibra utilizada no presente estudo, a Nutriose ${ }^{\circledR}$, é classificada como uma dextrina resistente, obtida através de amidos convencionais (não geneticamente modificados) do milho ou trigo que são submetidos a um processo denominado dextrinificação, que transforma sua estrutura molecular conferindo-Ihe o perfil de fibra alimentar, tornando-a fermentável e menos digerível e com potencial prebiótico. (LEFRANC-MILLOT, 2008; PASMAN et al., 2006).

Diante do exposto, o objetivo deste trabalho foi adicionar fibra de trigo em salsichas, que é um produto popular e amplamente consumido, avaliar sua aceitação sensorial e seus aspectos tecnológicos e verificar, através de uma avaliação subjetiva, se a adição dessa fibra poderia aumentar a sensação de saciedade do consumidor, buscando favorecer o consumo de fibras pela população e contribuindo no controle de obesidade.

\section{Material e métodos}

\subsection{Processamento das salsichas}

As matérias-primas de origem animal utilizadas foram carne de dianteiro bovino (60\%) e toucinho suíno sem couro $(17,3 \%)$. Os demais ingredientes foram: sal de cura (RS1, Fego Alimentos Ltda.) (0,1\%), mistura de condimentos contendo eritorbato de sódio e tripolifosfato de sódio (Topmix AR 01, Fego Alimentos Ltda.) (1\%). Como fibra solúvel, foi utilizada a Nutriose ${ }^{R}$ (Roquette Freres) cedida pela Labonathus Ltda., sua representante comercial no Brasil. Água (gelo) foi adicionada em cada formulação até completar 100\%.

Foram elaboradas três formulações de salsicha, uma amostra Controle (sem adição de Nutriose ${ }^{\mathrm{R}}$ ) e as demais com 1,5\% e $3 \%$ de adição da fibra. As salsichas foram formuladas de acordo com o que estabelece a legislação sobre os "Padrões de Identidade e Qualidade 
de Salsicha", com limites máximos ou mínimos para os teores de umidade (máx. =65\%), gordura (máx. =30\%), proteína (mín. = 12\%), amido (máx.-2\%) e carboidratos totais (máx. $=7 \%$ ) (BRASIL, 2000).

Os ingredientes foram pesados e colocados em homogeneizador de bacia rotativa (cutter) na sequência: as carnes, o sal, o condimento, o sal de cura, metade do gelo, o toucinho, o restante do gelo e a fibra, homogeneizando-os por aproximadamente $10 \mathrm{~min}$, sendo retiradas as emulsões antes de atingirem $14^{\circ} \mathrm{C}$. Estas foram embutidas em tripas celulósicas e levadas à estufa. O cozimento iniciou em temperatura de $60^{\circ} \mathrm{C}$ até completo avermelhamento das salsichas (reação de cura), por 30 minutos. Com vapor direto e a $70{ }^{\circ} \mathrm{C}$, foram cozidas por mais 30 minutos e, por fim, a $80^{\circ} \mathrm{C}$, até a temperatura interna atingir $72{ }^{\circ} \mathrm{C}$. As salsichas foram resfriadas em chuveiro durante 20 minutos, foram retiradas as tripas, embaladas a vácuo e acondicionadas sob refrigeração a $5^{\circ} \mathrm{C} \pm 1{ }^{\circ} \mathrm{C}$ até a realização das análises.

\subsection{Análises instrumentais}

\subsubsection{Estabilidade de emulsão}

A análise de estabilidade de emulsão foi realizada nas massas cárneas antes do cozimento, segundo o método de Parks \& Carpenter (1987).

\subsubsection{Rendimento do processo}

Para determinação do rendimento do processo, as salsichas, após embutidas, foram pesadas antes e depois do cozimento em estufa. O rendimento do processo foi calculado pela seguinte equação: Rendimento $(\%)=($ peso final/ peso inicial) x 100 .

\subsubsection{Valor de $\mathrm{pH}$}

Foram realizadas medições do pH em 10 amostras de cada formulação utilizando pHmetro com eletrodo de perfuração da marca Hanna, Modelo HI 99163, Woonsocket - USA, diretamente nas amostras.

\subsubsection{Cor objetiva}

A cor objetiva foi determinada com um colorímetro portátil Miniscan XE (HunterLab), fonte de luz de D65, ângulo de $10^{\circ}$ e abertura de célula de $30 \mathrm{~mm}$, na parte interna em salsichas. Utilizaram-se os padrões de cor do sistema CIELab: L* (luminosidade), a* (intensidade da cor vermelha-verde) e $b^{*}$ (intensidade da cor amarela-azul).

\subsubsection{Determinação de lipídios}

Para a análise de determinação de lipídios, foi utilizado o método de Bligh e Dyer (1959).

\subsubsection{Determinação de umidade}

A determinação de umidade foi realizada por secagem em estufa a $105^{\circ} \mathrm{C}$.

\subsubsection{Determinação de cinzas}

A determinação de conteúdo mineral (Cinzas) foi realizada por incineração em mufla a $550^{\circ} \mathrm{C}$ (CUNNIFF, 1997).

\subsubsection{Determinação de proteínas}

O teor de proteínas foi analisado pelo método de combustão (AOAC, 1968).

\subsubsection{Análise de perfil de textura}

A análise de perfil de textura instrumental (TPA) foi realizada em um texturômetro (TA-XT2i, Stable Micro Systems,) calibrado com peso padrão de $5 \mathrm{~kg}$. As 10 amostras de $2 \mathrm{~cm}$ de comprimento de cada tratamento foram submetidas a testes de compressão com a sonda cilíndrica de $30 \mathrm{~mm}$ do próprio equipamento, numa velocidade de $1 \mathrm{~mm} / \mathrm{min}$, até uma deformação de 50\% (BOURNE, 1982). As características estudadas foram dureza, elasticidade e mastigabilidade.

\subsection{Análise sensorial}

Para avaliação sensorial das salsichas, foi realizado teste afetivo de aceitação (MEILGAARD et al., 1999), com 60 consumidores regulares de salsicha, sendo 38 mulheres e 22 homens. As análises foram realizadas em Laboratório de Análise Sensorial em cabines individuais utilizando luz vermelha. As salsichas foram pré-aquecidas em água (3 minutos, $100^{\circ} \mathrm{C}$ ), cortadas em pedaços de $2 \mathrm{~cm}$ e mantidas aquecidas em banho-maria em estufa a $50{ }^{\circ} \mathrm{C}$ até o momento das análises. As amostras foram servidas aos provadores de forma monádica, seguindo delineamento de blocos completos aleatorizados, em recipientes codificados com algarismos de 3 dígitos aleatórios, acompanhadas de água e biscoitos de água e sal. Foi solicitado aos consumidores que avaliassem as amostras em relação aos atributos sabor, textura, suculência e aceitação global, utilizando escala hedônica de nove pontos (1- Desgostei muitíssimo; 9- Gostei muitíssimo).

\subsection{Análise de saciedade}

Para a análise de saciedade, 30 consumidores regulares de salsichas receberam 3 pacotes codificados com algarismos de 3 dígitos aleatórios. Cada pacote continha 2 salsichas com aproximadamente 50 g cada, 1 para cada formulação. As amostras foram consumidas monadicamente durante 3 dias seguidos, sendo um tratamento a cada dia. A ordem de consumo variou para cada consumidor, seguindo delineamento de blocos 
completos aleatorizados. A forma de preparo e consumo foi explicada pessoalmente e também descrita na ficha explicativa entregue aos provadores com a seguinte informação: "Você está recebendo 3 amostras de salsicha e deverá consumi-las em dias diferentes na ordem descrita. Aquela que você for consumir, cozinhe por 3 minutos em água fervente. Você não poderá consumir quaisquer outros alimentos durante as 2 horas de teste, mas poderá consumir a bebida que desejar, contanto que repita a mesma bebida e a mesma quantidade para as 3 amostras, e deverá anotar nas escalas o quanto você está sentindo de fome em cada horário solicitado". Para a anotação da sensação de fome/saciedade, os consumidores receberam mais 3 fichas (uma para cada dia/tratamento) com seis Escalas Visuais Analógicas por ficha, sendo uma escala para cada tempo, em que o tempo zero $(\mathrm{t}=0)$ referia-se à sensação de fome antes do consumo, o tempo "um" ( $t=1)$, à fome imediatamente após o consumo e os tempos seguintes, a cada 30 minutos, até completarem 2 horas de teste $(t=5)$. A distância da extremidade esquerda da Escala Visual Analógica até a marca assinalada pelos consumidores foi medida posteriormente com auxílio de uma régua pelos pesquisadores. O valor obtido em cada uma das escalas para a sensação de fome variou de 0 a 10. O Termo de Consentimento Livre e Esclarecido foi assinado por todos os voluntários dos testes de aceitação sensorial e de saciedade. O estudo foi submetido ao Comitê de Ética em Pesquisa da Universidade (CAAE:13354113.3.0000.5422).

\subsection{Análise estatística}

As salsichas foram processadas seguindo um delineamento inteiramente casualizado com 3 tratamentos e 2 repetições do processamento de cada tratamento. Os dados obtidos foram tratados com o programa estatístico SAS ${ }^{\circledR}$ (Statistic Analisy System), versão 8.02, por análise de variância ANOVA e teste de Tukey, ao nível de $5 \%$ de confiança. Para os testes de aceitação sensorial e de saciedade subjetiva, consideraram-se no modelo os efeitos de tratamento e de consumidor. Para as análises estatísticas dos resultados do teste de saciedade, os dados foram separados em etapas, em função das especificidades destas etapas: primeiramente foram comparadas isoladamente, por ANOVA e teste de Tukey, as médias da sensação de fome dos provadores antes do consumo (TO) e logo após o consumo das salsichas (T1). O comportamento da sensação de fome, após 30 minutos (T2) e até o final dos 120 minutos (T5), foi analisado conjuntamente para as variáveis tratamento, tempo e interação tempo $X$ tratamento, utilizando-se o procedimento proc mixed do $\mathrm{SAS}^{\circledast}$, sendo submetidas a ajustes de modelos de regressão linear para verificar a tendência de comportamento das amostras.

\section{Resultados e discussões}

Os resultados das análises físico-químicas (parâmetros tecnológicos) da salsicha com adição de fibra de trigo estão apresentados na Tabela 1.

Exceto para a luminosidade $\left(L^{*}\right)$, não houve diferenças entre as formulações com adição de fibras e a formulação Controle (sem fibras) em nenhum dos parâmetros tecnológicos analisados.

Os teores de umidade não diferiram entre amostras, porém percebeu-se uma discreta diminuição conforme o aumento da quantidade de fibra adicionada, que pode ter ocorrido em função da adição da fibra ter substituído parte da água na formulação. Estes resultados foram bem próximos aos encontrados por Garcia-Cruz et al. (1996), que produziram salsichas de carne bovina com adição

Tabela 1. Médias \pm erro padrão dos resultados das análises físico-químicas das salsichas Controle e com adição de $1,5 \%$ e $3 \%$ de fibra.

\begin{tabular}{|c|c|c|c|c|c|c|}
\hline \multirow{2}{*}{$\begin{array}{c}\text { Parâmetros físico-químicos } \\
\text { Umidade (\%) }\end{array}$} & \multicolumn{2}{|c|}{ Controle } & \multicolumn{2}{|c|}{$1,5 \%$ de Fibra } & \multicolumn{2}{|c|}{$3 \%$ de Fibra } \\
\hline & $62,4^{a}$ & $\pm 0,2$ & $60,1^{a}$ & $\pm 0,8$ & $59,2^{\mathrm{a}}$ & $\pm 1,8$ \\
\hline Cinzas (\%) & $3,2^{\mathrm{a}}$ & $\pm 0,2$ & $3,4^{a}$ & $\pm 0,3$ & $3,3^{a}$ & $\pm 0,3$ \\
\hline Proteína (\%) & $16,9^{a}$ & $\pm 0,9$ & $18,4^{\mathrm{a}}$ & $\pm 0,4$ & $16,8^{a}$ & $\pm 0,8$ \\
\hline Lipídios (\%) & $16,7^{a}$ & $\pm 0,9$ & $16,9^{a}$ & $\pm 0,8$ & $16,5^{a}$ & $\pm 0,7$ \\
\hline pH & $6,1^{\mathrm{a}}$ & $\pm 0,3$ & $6,1^{\mathrm{a}}$ & $\pm 0,2$ & $6,0^{\mathrm{a}}$ & $\pm 0,1$ \\
\hline Estabilidade emulsão (\%) & $15,1^{\mathrm{a}}$ & $\pm 0,3$ & $15,7^{\mathrm{a}}$ & $\pm 0,01$ & $15,1^{\mathrm{a}}$ & \pm 0 \\
\hline Rendimento & $83,7^{a}$ & $\pm 0,3$ & $84,4^{a}$ & $\pm 1,2$ & $82,4^{a}$ & $\pm 1,2$ \\
\hline Dureza (g) & $7238,6^{a}$ & $\pm 1566,6$ & $7004,1^{\mathrm{a}}$ & $\pm 1594,6$ & $6355,5^{a}$ & $\pm 2461,2$ \\
\hline Elasticidade (razão) & $0,87^{a}$ & $\pm 0,02$ & $0,89^{a}$ & $\pm 0,02$ & $0,88^{a}$ & $\pm 0,01$ \\
\hline Mastigabilidade (g) & $4332,1^{a}$ & $\pm 1545,2$ & $4434,2^{\mathrm{a}}$ & $\pm 1525,3$ & $3546,7^{a}$ & $\pm 1796,1$ \\
\hline $\mathbf{L}^{*}$ & $57,6^{\mathrm{ab}}$ & $\pm 0,04$ & $58,6^{a}$ & $\pm 0,02$ & $56,6^{b}$ & $\pm 0,7$ \\
\hline$a^{*}$ & $11,8^{a}$ & $\pm 0,01$ & $11,5^{\mathrm{a}}$ & $\pm 0,04$ & $11,8^{a}$ & $\pm 0,6$ \\
\hline $\mathbf{b}^{*}$ & $11,8^{a}$ & $\pm 0,02$ & $11,7^{\mathrm{a}}$ & $\pm 0,02$ & $11,8^{a}$ & $\pm 0,1$ \\
\hline
\end{tabular}

$\left(L^{*}\right)$ Luminosidade, $\left(a^{*}\right)$ intensidade da cor vermelha e $\left(b^{*}\right)$ intensidade da cor amarela; Letras diferentes na mesma linha indicam diferença significativa $(p<0,05)$. 
de goma guar e goma xantana na proporção de 1\% em substituição parcial ao amido de mandioca. Os teores de umidade da salsicha com adição de fibra de trigo atenderam aos padrões da legislação que estabelece teores de umidade máximos de 65\% para salsicha (BRASIL, 2000).

A quantidade de cinza encontrada foi próxima à encontrada por Bortoluzzi (2009), que estudou os efeitos da adição de fibras de laranja, trigo e beterraba e encontrou valores de resíduo mineral entre 2,6\% e 3,5\%. Não há teores de cinzas expressos nos rótulos nem limites definidos na legislação, porém a cinza é constituída principalmente de $\mathrm{K}, \mathrm{Na}$, Ca e Mg e um percentual elevado de cinzas pode estar relacionado com uma quantidade de sal maior do que o necessário, o que não ocorreu neste presente estudo.

Os resultados para os teores de proteínas e de gordura atenderam ao que estabelece a Instrução Normativa (BRASIL, 2000) para esse tipo de produto que não deve conter teores de proteínas inferiores a $12 \%$ ou 6 gramas por porção de 50 gramas de salsicha e gordura de no máximo 30\%.

Com a adição da fibra de trigo nas salsichas, os resultados de $\mathrm{pH}$ ficaram próximos aos estudados por Barreto (2007) em mortadelas de carne bovina com adição de 5\% das fibras de trigo, inulina e fibra de aveia e não diferiram da amostra Controle. Huang et al. (2011) produziram salsichas com adição das fibras de trigo, de aveia e inulina e encontraram resultados para $\mathrm{pH}$ entre 6,5 e 6,6, e que também não diferiram da amostra Controle (sem adição de fibras).

A salsicha é uma emulsão de carne e as análises de estabilidade de emulsão e rendimento do processo são importantes parâmetros de qualidade. Seria possível esperar uma redução da porcentagem de perda de exsudado devido à incorporação da fibra. Segundo Barreto (2007), as fibras possuem alta capacidade de retenção de água, podendo auxiliar na melhoria da estabilidade de emulsão e rendimento do processo. No entanto, não ocorreu, no presente estudo, diferença entre as salsichas com adição de fibra de trigo e a Controle (sem fibra) na estabilidade da emulsão.

De acordo com Roça (2000), isso depende de diversos fatores como a estrutura química dos componentes da fibra, tipo de parede celular da qual foi obtida, anatomia e tamanho das partículas e sua porosidade, $\mathrm{pH}$, temperatura e processamento e também de fatores como tamanho das partículas de gordura da emulsão, quantidade e tipo de proteína e a própria viscosidade da emulsão. Na análise do perfil de textura instrumental (TPA) também não se verificaram diferenças significativas em nenhum dos parâmetros avaliados (dureza, elasticidade e mastigabilidade).

Huang et al. (2011) produziram salsichas de carne de porco com adição de 3,5\% e 7\% das fibras de trigo, aveia e inulina e encontraram valores de dureza de 218,22, observado na salsicha com adição de 3,5\% de inulina, 238,87 para a formulação Controle, 457,82 para a formulação com 7\% de fibra de aveia, e 670,66 para a formulação com acréscimo de $7 \%$ de fibra de trigo, mostrando que, com exceção da inulina que teve valor menor que a Controle, nas demais os valores foram maiores, conferindo maior firmeza para salsichas com acréscimo de fibra de aveia e de trigo, divergindo dos resultados do presente estudo. Guerreiro (2006) relata que prováveis causas de dureza excessiva em produtos emulsionados cárneos são o teor muito elevado de carnes magras ou tecido conjuntivo e adição insuficiente de água, o que provavelmente deve ter ocorrido no presente estudo. O estudo de Barreto (2007), com adição de 4\% de fibra de trigo em mortadela de frango, apresentou valor 0,88 para elasticidade, e nas porcentagens de $2 \%$ de fibra de trigo e Controle (sem fibras), 0,87 e 0,91, respectivamente, muito próximos aos encontrados neste estudo.

Na luminosidade, a amostra com 1,5\% de fibra apresentou um valor maior que a amostra com 3\%, mas ambas não diferiram da amostra Controle $(p<0,05)$. Embora tenha havido essa diferença, os resultados ficaram bem próximos aos encontrados por Ferracioli (2012), que avaliou qualidades de salsichas do tipo hot dog de marcas comerciais, observando valores entre 55,8 e 58,0 para o parâmetro $L^{*}$.

\subsection{Análise sensorial}

Os resultados do teste de aceitação sensorial das salsichas com ou sem fibra estão representados na Tabela 2.

Os consumidores avaliaram de maneira similar o sabor das três diferentes salsichas, ficando entre "gostei moderadamente" e "gostei muito". Algumas fibras podem causar alterações de sabor e impressão global, porém a fibra solúvel de trigo possui um sabor neutro não causando essa diferença entre as três formulações.

Na avaliação de aceitação sensorial para o atributo textura, as notas ficaram próximas do conceito "Gostei moderadamente", e também não diferiram entre si. Já para

Tabela 2. Média \pm erro padrão dos resultados de aceitação sensorial de salsicha com adição de fibra.

\begin{tabular}{lcccccc}
\multicolumn{1}{c}{ Atributo } & \multicolumn{2}{c}{ Controle } & $\begin{array}{c}\text { 1,5\% Fibra de } \\
\text { trigo }\end{array}$ & \multicolumn{2}{c}{$\begin{array}{c}\text { 3\% Fibra de } \\
\text { trigo }\end{array}$} \\
\hline Sabor & $7,6^{\mathrm{a}}$ & $\pm 1,3$ & $7,2^{\mathrm{a}}$ & $\pm 1,5$ & $7,1^{\mathrm{a}}$ & $\pm 1,5$ \\
Textura & $6,9^{\mathrm{a}}$ & $\pm 1,7$ & $6,8^{\mathrm{a}}$ & $\pm 1,7$ & $7,1^{\mathrm{a}}$ & $\pm 1,7$ \\
$\begin{array}{l}\text { Suculência } \\
\text { Aceitação }\end{array}$ & $7,5^{\mathrm{ab}}$ & $\pm 1,3$ & $7,1^{\mathrm{b}}$ & $\pm 1,7$ & $7,7^{\mathrm{a}}$ & $\pm 1,4$ \\
global & $7,5^{\mathrm{ab}}$ & $\pm 1,5$ & $7,1^{\mathrm{b}}$ & $\pm 1,5$ & $7,6^{\mathrm{a}}$ & $\pm 1,5$ \\
\hline
\end{tabular}

Letras diferentes na mesma linha indicam diferença significativa a $\mathrm{p}<0,05$. 
os atributos "suculência" e "aceitação global", a salsicha com $3 \%$ de fibra mostrou-se mais aceita $(p<0,05)$ que a com $1,5 \%$, sendo que ambas não diferiram da Controle. Uma característica favorável das fibras solúveis é que em contato com a água formam um gel viscoso e quando adicionadas em alimentos pode deixá-los mais suculentos. Garcia-Cruz et al. (1996) também obteve boas médias entre 6 e 7 para este atributo do teste de aceitação em salsichas elaboradas com adição de 1\% das gomas guar e xantana em substituição parcial ao amido.

\subsection{Análise de saciedade}

Os resultados do teste de saciedade estão representados na Figura 1.

As médias atribuídas pelos consumidores para a sensação de fome no T0 indicaram que eles sentiam fome antes do teste e não apresentaram diferença entre as três amostras. Esse resultado era desejado e importante, pois, se as sensações de fome iniciais tivessem diferido, poderia interferir nos resultados dos tempos seguintes. Alguns autores preferem determinar um tempo de jejum igual para todos os consumidores antes das análises de saciedade para que uma provável diferença antes do teste não venha interferir nas sensações de fome seguintes, como fez Menezes et al. (2011) que testaram a saciedade da farinha de banana verde em voluntários que permaneceram em jejum durante 4 horas antes do teste. No presente estudo, não foi solicitado um período específico de jejum, mas apenas orientado aos consumidores que realizassem o teste no intervalo entre duas principais refeições diárias.

No T1 (logo após o consumo), a média da sensação de fome teve uma queda brusca para todas as amostras e também não houve diferença entre elas. Isso demonstra que todas as salsichas saciaram igualmente a fome imediatamente após serem consumidas. Esse fato pode ser explicado devido à matéria-prima principal da salsicha ser a carne, e esta, por sua vez, é digerida no estômago e permanece ali por mais tempo, ocasionando esvaziamento gástrico demorado, causando a sensação de saciedade, independentemente de conterem fibras ou não.

Os resultados entre o T2 (30 minutos após consumo) e T5 (120 minutos após consumo) não apresentaram diferenças entre os tratamentos nem na interação tempo $X$ tratamento, porém houve um aumento significativo da sensação de fome ao longo do tempo, em que o comportamento de aumento da sensação de fome foi semelhante para todas as amostras e pode ser bem explicado por um polinômio linear (Figura 2).

Quanto ao tipo de teste aplicado neste estudo, este se mostrou eficiente, pois, segundo ele, houve um retorno da fome ao longo do tempo, que é o que naturalmente se espera que ocorra após a ingestão de um alimento. No entanto, nas condições testadas, o consumo das salsichas com adição de 1,5\% e 3\% da fibra de trigo não contribuiu para aumentar significativamente $(p>0,05)$ a sensação de saciedade nem para retardar o retorno da fome.

Um ponto importante a ser considerado é a quantidade da fibra adicionada. Encontrar a proporção correta que venha causar saciedade, sem, porém, interferir nos aspectos tecnológicos do produto constitui um desafio. Cani et al. (2006) testaram, com 10 provadores selecionados, a ingestão diária de $16 \mathrm{~g}$ (8 g no café da manhã e $8 \mathrm{~g}$ no jantar) da fibra oligofrutose durante 2 semanas, quantificando a ingestão de alimentos/calorias ingeridos por eles. Estes autores concluíram que essa fibra, na proporção consumida, aumentou a saciedade. Guérin-Deremaux et al. (2011) verificaram que a adição de 8 a 24 gramas de fibra de trigo em suco de laranja

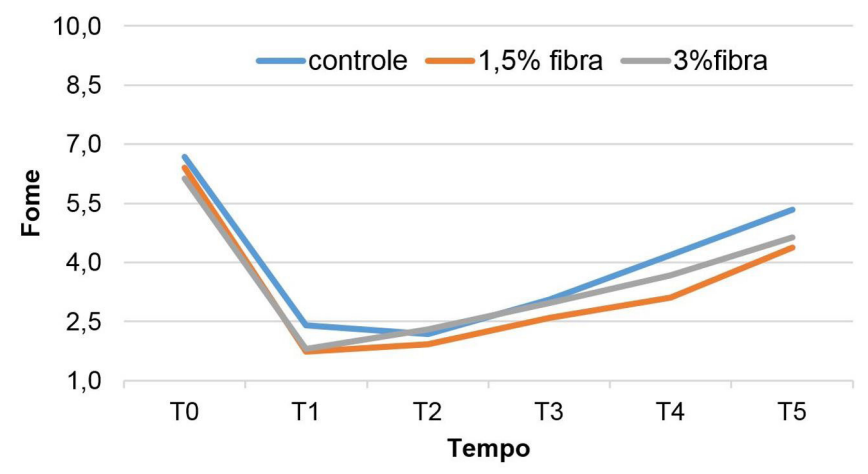

Figura 1. Gráfico de perfis médios de fome por formulação ao longo do tempo. (T0) = antes de comer; (T1) = logo após comer; $(\mathrm{T} 2)=1 / 2$ hora após comer; $(\mathrm{T} 3)=1$ hora após comer; $(\mathrm{T} 4)=1 \frac{1}{2}$ horas após comer; $(\mathrm{T} 5)=2$ horas após comer.

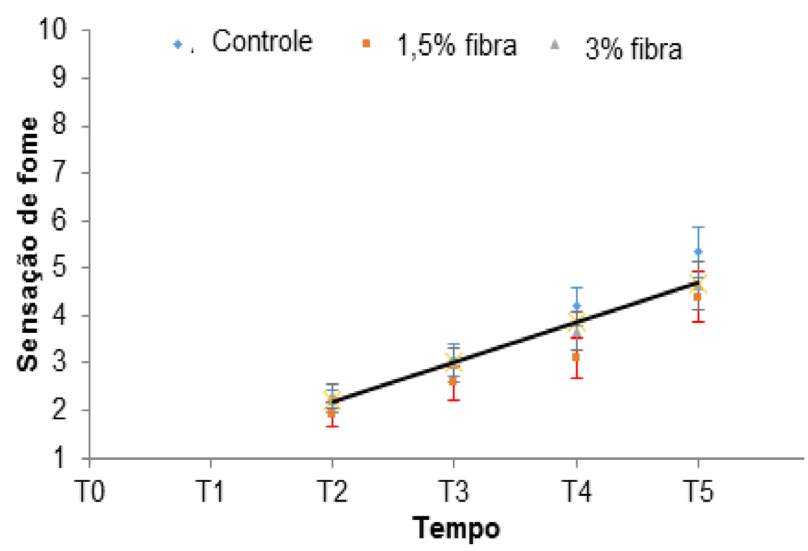

Figura 2. Reta representativa da sensação de fome nos consumidores entre 30 e 120 minutos após o consumo das salsichas com ou sem adição de fibra de trigo. (T0) = antes de comer; (T1) = logo após comer; (T2) = 1/2 hora após comer; $($ T3 $)=1$ hora após

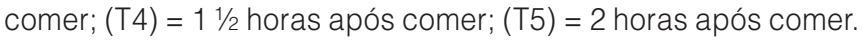


Saciedade subjetiva, aceitação sensorial e aspectos tecnológicos de salsicha com adição de fibra de trigo Borrajo, K. H. T. et al.

aumentou a saciedade e retardou a volta da fome nos provadores. No caso deste presente estudo, as quantidades de 1,5\% e 3\% desta mesma fibra, quando adicionadas às salsichas, não alteraram significativamente a sensação de fome dos consumidores. Possivelmente, o consumo de somente 1,5 e $3 \mathrm{~g}$ de fibra na refeição (duas salsichas de $50 \mathrm{~g}$ ) não tenha sido suficiente para demonstrar resultados positivos.

O tempo de teste também deve ser analisado, pois, no último tempo (T5), após 2 horas da ingestão das salsichas com fibras, os resultados demonstraram que os provadores relataram nas EVAS que sentiam menos fome ao comerem a salsicha com 3\%, com média de quase 1 ponto de diferença, em comparação à média de quando comeram as salsichas sem fibras, ou seja, foi percebido que a saciedade no tratamento Controle foi menor, porém não foi significativa. É possível que, em um intervalo de tempo maior, poderiam ter sido observadas diferenças significativas na sensação de fome/saciedade.

Essa hipótese tem fundamento, considerando outro mecanismo de saciedade que não o do esvaziamento gástrico demorado, mas sim da fermentação da Nutriose ${ }^{R}$, que, ao chegar ao intestino, é caracteristicamente lenta, o que resulta em uma lenta absorção dos ácidos graxos de cadeia curta (LEFRANC-MILLOT, 2008; PASMAN et al., 2006) e, uma vez que esses são utilizados como fonte de energia pelo organismo, a fibra de trigo pode impactar positivamente as questões de saciedade.

\section{Conclusões}

Mesmo não tendo sido observada maior sensação de saciedade pelo consumo das salsichas com adição de fibra de trigo, os resultados do presente estudo demonstraram que salsichas adicionadas com 1,5\% e $3 \%$ desta fibra podem ser produzidas, sem prejuízo das características tecnológicas e com boa aceitação sensorial, podendo favorecer o aumento do consumo de fibras pela população.

\section{Referências}

ASSOCIATION OF OFFICIAL ANALYTICAL CHEMISTS - AOAC. AOAC Official Method 968.06: protein (Crude) in animal feed. Dumas method. Journal of of AOAC International, Washington, v. 51, p. $766,1968$.

BARRETO, A. C. S. Efeito da adição de fibras como substitutos de gordura em mortadela. 2007. 163 f. (Doutorado em Tecnologia de Alimentos)-Universidade Estadual de Campinas, Campinas, 2007.

BIRKETVEDT, G. S.; SHIMSHI, M.; ERLING, T.; FLORHOLMEN, $J$. Experiences with three different fiber supplements in weight reduction. Medical Science Monitor, New York, v. 11, n. 1, p. 5-8, 2005.
BLIGH, E. G.; DYER, W. J. A rapid method of total lipid extraction and purification. Canadian Journal of Biochemistry and Physiology, Ottawa, v. 37, n. 8, p. 911-917, 1959. http://dx.doi. org/10.1139/059-099. PMid:13671378.

BORTOLUZZI, R. C. Aplicação da fibra obtida da polpa de laranja na elaboração de mortadela de frango. 2009. $83 \mathrm{f}$. Tese (Doutorado em Ciências dos Alimentos)-Faculdade de Ciências Farmacêuticas, Universidade de São Paulo, São Paulo, 2009. http://dx.doi.org/10.11606/T.9.2009.tde-13072009-214817.

BOURNE, M. C. Food texture and viscosity: concept and measurement. New York: Academic Press, 1982.

BRASIL. Ministério da Agricultura, Pecuária e Abastecimento. Instrução Normativa $n^{\circ} 4$, de 31 de março de 2000. Aprova os Regulamentos Técnicos de identidade e qualidade de carne mecanicamente separada, de mortadela, de lingüiça e de salsicha. Diário Oficial [da] República Federativa do Brasil, Brasília, DF, 5 abr. 2000.

CANDOGAN, K.; KOLSARICI, N. Store stability of low-fat beef frankfurters formulated with carrageen or carrageen with pectin. Meat Science, Barking, v. 64, n. 2, p. 207-214, 2003. http:// dx.doi.org/10.1016/S0309-1740(02)00182-1. PMid:22062868.

CANI, P. D.; JOLY, E., HORSMANS, Y., DELZENNE, N. M. Oligofructose promotes satiety in healthy human: a pilot study. European Journal of Clinical Nutrition, London, v. 60, n. 5 , p. 567-572, 2006.

CUNNIFF, P. (Ed.). Official methods of analysis of the Association of Official Analytical Chemists. 16th ed. Washington: AOAC, 1997.

FERRACIOLI, V. R. Avaliação da qualidade de salsichas do tipo Hot Dog durante o armazenamento. 2012. $115 \mathrm{f}$. Dissertação (Mestrado em Engenharia de Processos Químicos e Bioquímicos)-Centro universitário do Instituto Mauá de Tecnologia, São Paulo, 2012.

FLINT, A.; RABEN, A.; BLUNDELL, J.; ASTRUP, A. Reproducibility, power and validity of visual analogue scales in assessment of appetite sensations in single test meal studies. International Journal of Obesity and Related Metabolic Disorders, Hampshire, v. 24, n. 1, p. 38-48, 2000. http://dx.doi.org/10.1038/sj.ijo.0801083. PMid:10702749.

GARCIA-CRUZ, C. H.; SCAMPARINI, A. R. P.; HOFFMANN, F. L. Elaboração de salsichas utilizando goma guar e goma xantana em substituição ao amido de mandioca (Manihot esculenta). Alimentos e Nutrição, Araraquara, v. 7, n. 1, p. 25-35, 1996.

GUÉRIN-DEREMAUX, L.; POCHAT, M.; REIFER, C.; WILS, D.; CHO, S.; MILLER, L. E. The soluble fiber NUTRIOSE® induces a dose-dependent beneficial impact on satiety over time in humans. Nutrition Research, New York, v. 31, n. 9, p. 665-672, 2011. http://dx.doi.org/10.1016/j.nutres.2011.09.004. PMid:22024490. 
Saciedade subjetiva, aceitação sensorial e aspectos tecnológicos de salsicha com adição de fibra de trigo Borrajo, K. H. T. et al.

GUERREIRO, L. Produção de salsicha. Rio de Janeiro: Redetec, 2006. p. 22. Dossiê técnico. Disponível em: <http://sbrt.ibict.br/ dossie-tecnico?dossie=MzA>. Acesso em: 3 out. 2015.

HALPERN, Z. S. C.; RODRIGUES, M. D. B.; COSTA, R F D. Determinantes fisiológicos do controle de peso e apetite. Revista de Psiquiatría Clínica, Santiago, v. 31, n. 4, p. 150-153, 2004.

HUANG, S. C.; TSAI, Y. F.; CHEN, C. M. Effects of wheat fiber, oat fiber, and inulin on sensory and physico-chemical properties of chinese-style sausages. Asian-Australasian Journal of Animal Sciences, Seoul, v. 24, n. 6, p. 875-880, 2011. http:// dx.doi.org/10.5713/ajas.2011.10317.

INSTITUTO BRASILEIRO DE GEOGRAFIA E ESTATÍSTICA IBGE. Pesquisa de orçamentos familiares 2008-2009: análise do consumo alimentar pessoal no Brasil. Rio de Janeiro, 2011. Disponível em: <http://www.ibge.gov.br/home/ estatistica/ populacao/condicaodevida/pof/2008_2009_analise_consumo/ pofanalise_2008_2009.pdf>.

INUI, A.; ASAKAWA, A.; BOWERS, C. Y.; MANTOVANI, G.; LAVIANO, A.; MEGUID, M. M.; FUJIMIYA, M. Ghrelin, appetite, and gastric motility: the emerging role of the stomach as an endocrine organ. The FASEB Journal, Bethesda, v. 18, n. 3, p. 439-456, 2004. http://dx.doi.org/10.1096/fj.03-0641rev. PMid:15003990.

LEFRANC-MILLOT, C. NUTRIOSE® 06: a useful soluble dietary fibre for added nutritional value. Nutrition Bulletin, London, v. 33, n. 3, p. 234-239, 2008. http://dx.doi.org/10.1111/j.14673010.2008.00711.x.

LIU, S.; STAMPFER, M. J.; HU, F. B.; GIOVANNUCCI, E.; RIMM, E.; MANSON, J. E.; HENNEKENS, C. H.; WILLETT, W. C. Wholegrain consumption and risk of coronary heart disease: results from the Nurses' Health study. The American Journal of Clinical Nutrition, Bethesda, v. 70, n. 3, p. 412-419, 1999. PMid:10479204.

MEILGAARD, M. C.; CARR, B. T.; CIVILLE, G. V. Sensory evaluation techniques. 3. ed. Boca Raton: CRC Press, 1999.

MENEZES, E.W.; DAN, M.C.T.; GIUNTINI, E. B.; FUKUMORI, C.; LAJOLO, F. M. Efeito do consumo de farinha de banana verde sobre o perfil de hormônios gastrintestinais. Revista Nutrire, São Paulo, v. 36, p. 67, 2011. Suplemento.
MONTONEN, J.; KNEKT, P.; JARVINEN, R.; AROMAA, A.; REUNANEN, A. Whole-grain and fiber intake and the incidence of type 2 diabetes. The American Journal of Clinical Nutrition, Bethesda, v. 77, n. 3, p. 622-629, 2003. PMid:12600852.

NAZARE, J. A.; SAUVINET, V.; NORMAND, S.; GUÉRIN-DEREMAUX, L.; GABERT, L.; DÉSAGE, M.; WILS, D.; LAVILLE, M. Impact of a resistant dextrin with a prolonged oxidation pattern on day-long ghrelin profile. Journal of the American College of Nutrition, New York, v. 30, n. 1, p. 63-72, 2011. http://dx.doi.org/10.1080 /07315724.2011.10719945. PMid:21697540.

PARKS, L. L.; CARPENTER, J. A. Functionality of six nonmeat proteins in meat emulsion systems. Journal of Food Science, Mysore, v. 52, n. 2, p. 271-274, 1987. http://dx.doi. org/10.1111/j.1365-2621.1987.tb06590.x.

PASMAN, W.; WILS, D.; SANIEZ, M. H.; KARDINAAL, A. Longterm gastrointestinal tolerance of NUTRIOSE ${ }^{\circledR} \mathrm{FB}$ in healthy men. European Journal of Clinical Nutrition, London, v. 60, n. 8, p. 1024-1034, 2006. http://dx.doi.org/10.1038/sj.ejcn.1602418. PMid:16482066

PETRUZZIELLO, L.; IACOPINI, F.; BULAJIC, M.; SHAH, S.; COSTAMAGNA, G. Review article: uncomplicated diverticular disease of the colon. Alimentary Pharmacology \& Therapeutics, Oxford, v. 23, n. 10, p. 1379-1391, 2006. http://dx.doi.org/10.1111/ j.1365-2036.2006.02896.x. PMid:16669953.

RANINEN, K.; LAPPI, J.; MYKKÄNEN, H.; POUTANEN, K. Dietary fiber type reflects physiological functionality: comparison of grain fiber, inulin, and polydextrose. Nutrition Reviews, New York, v. 69, n. 1, p. 9-21, 2011. http://dx.doi.org/10.1111/j.17534887.2010.00358.x. PMid:21198631.

ROÇA, R. O. Tecnologia da carne e produtos derivados. Botucatu: UNESP, 2000. 202 p.

WANG, Y.; MONTEIRO, C.; POPKIN, B. M. Trends of obesity and underweight in older children and adolescents in the United States, Brazil, China, and Russia. The American Journal of Clinical Nutrition, Bethesda, v. 75, n. 6, p. 971-977, 2002. PMid:12036801. 